\title{
Investigation of Human Kinetic Energy Harvesting from Human Foot Strike
}

\author{
Joo Ann Teh and Zuraini Dahari* \\ School of Electrical and Electronic Engineering, Universiti Sains Malaysia, \\ Engineering Campus, 14300 Nibong Tebal, Pulau Pinang, Malaysia \\ *Corresponding author: eezuraini@usm.my
}

Published online: 15 April 2018

To cite this article: Joo Ann Teh and Zuraini Dahari. (2018). Investigation of human kinetic energy harvesting from human foot strike. Journal of Engineering Science, 14: 1-14, https://doi.org/10.21315/jes2018.14.1.

To link to this article: https://doi.org/10.21315/jes2018.14.1

\begin{abstract}
Due to the increasing demand of wireless sensor and low power consumer electronic device demand, energy harvesting from human motion become more favoured in recent years. In this study, an energy harvester based on human foot strike is proposed. The kinetic energy is harvested by using the piezoelectric materials and converted into an electrical energy. The effect of different types and different circuit configurations of piezoelectric materials are investigated in this study. Besides, the effect of several factors such as human body weight, gender and walking speed are also investigated. It is found that the MCFT-41T-1.0A1-141 piezoelectric ceramic which is thinner and greater in diameter can generate the greatest amount of electrical energy, which is $30.97 \mu \mathrm{W}$, whereas the best configuration is series connection. Besides, the electrical energy generated by people with greater body weight and faster walking speed are also higher. Finally, results also showed that a male can generate greater amount of electrical energy when compared to female.
\end{abstract}

Keywords: Energy harvesting, piezoelectric, human foot strike, electrical energy production

\section{INTRODUCTION}

In order to overcome resource depletion and pollution problem, researchers seek an alternative way to harness energy from renewable energy sources such as solar, wind, geothermal and hydropower. ${ }^{1,2}$ Unlike fossil fuel, these energy sources are replenished in a short period of time, which means that they will never run out. Renewable energy sources are also more environmental friendly. However, this type of renewable energy resources are vulnerable to weather and climate 
change. ${ }^{2}$ Since the weather and climate change is unpredictable and inconsistent, an alternate solution should be proposed in order to solve this problem.

Human body is an alternative source of energy. It contains enormous quantities of energy which can be used to fuel the movement and essential body functions. However, most of the energy are wasted as heat and other types of energy. These wasted energy could be captured and turned into electricity. Harvesting energy from human body is more reliable than harvesting energy from natural resources. This is because the energy generated from human body will not be easily affected by the location and the weather. Nowadays, many technologies has been invented in order to harness energy from human body. Walking is a common activity in our daily life. In 2004, studies found that the average steps per day

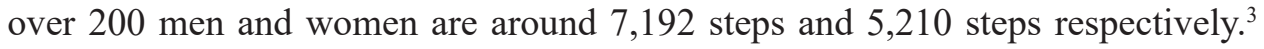
Therefore, the kinetic energy produced during walking or running are suitable to be harvested into an electrical energy.

In the previous researches, there are several techniques which are used to harvest the energy from human foot strike. In 2001, Shenck and Paradiso from Massachusetts Institute of Technology (MIT) laboratory have proposed a system to convert a low frequency piezoelectric shoe signals into a continuous and reliable energy source. ${ }^{4}$ This is done by embedding a flexible piezoelectric materials and a simple mechanical structure into the shoes. The harvester model was built from the lead zirconate titanate, which is also known as PZT dimorph which made up of two back-to-back, single sided unimorphs is used to harvest the energy from the heel strike while the 16 layer laminated multilaminar polyvinylidene fluoride (PVDF) bimorph stave mounted under the toes and midsole is used to harvest the energy from the bending of the ball of the foot. The average output power generated by the harvester is on the order of $10 \mathrm{~mW}$.

Another research about harvesting energy from foot strike was also proposed by Xie and Cai. ${ }^{5}$ In this research, a piezoelectric energy harvester which consist of an amplification mechanism was introduced. When a person applied a foot strike on the V-slider, it causes the two symmetrically distributed H-sliders to produce a horizontal force, which will be delivered to the com-like clamper later. Since there is a static framework supported at the mid-points of the clamped bimorphs, this results in a symmetrically deformation in the PZT bimorph. In this harvester, the piezoelectric bimorphs deform in 31 mode causes a large mechanical strain to produce and hence a high efficiency of output power can be achieved. This harvester can provide a power density of $0.41 \mathrm{~mW} / \mathrm{cm}^{3}$ at a frequency of $1.0 \mathrm{~Hz}$. 
The piezoelectric works based on the piezoelectricity effect. Piezoelectricity effect states that when a mechanical stress is applied on a dielectric material, electrical charges will be generated across their boundaries. ${ }^{6}$ There are two mode which are commonly be used to distinguish the transduction mechanism of piezoelectric, which are 31 and 33 mode. In 33 mode, the direction of the electric polarisation is same as the direction of the applied stress while in 31 mode, the direction of the electric polarisation is perpendicular to the direction of the applied stress.

One of the piezoelectric material which is available in the market is the PZT piezoelectric material. From the previous research, a study is carried out to investigate the factors that may influence the electric voltage generation of PZT. ${ }^{7}$ In the first experiment, the effect of the loading, frequency and number of PZT ceramic plates on the electric voltage generation are investigated. The results shows that as the loading value and the number of PZT ceramic plate increases, the maximum electric voltage also increases. However, the electric voltage is the highest when four PZT ceramic plate are used. The electric voltage decreases when the number of PZT ceramic plate is more than four. This is because the rigidity of the test samples increases when the number of PZT ceramic plate increases. When the number of PZT ceramic plate increases, the tensile strain is generated in the lower part of the PZT ceramic plate. The increase in the tensile strain results in the reduction of the electric voltage generation. Besides, the increase in frequency also increases its electric voltage generation. In the second experiment, the effect of stress area on the electric voltage generation is investigated. The electric voltage generated increases with the applied stress area.

Since the stress applied to the piezoelectric material is one the factor that will affect the electrical energy generated from the piezoelectric material, it is important to understand the foot pressure distribution of human. In year 2014, Deepashini and his group presented a review of literature on plantar pressure distribution and factor that may affect the plantar pressure. ${ }^{8}$ There are several factors that may affect the foot pressure distribution such as gender, body weight and walking speed. The research shows that the foot contact area and pressure distribution of men are greater than women. However, the foot contact area and pressure distribution in the midfoot between genders has no differences. Besides, as body weight increases, the total contact area and the peak plantar pressure of the foot also increases. Furthermore, as the walking speed increases, the mean peak pressure under the heel, central, metatarsals and toes increases while the peak pressure under the midfoot and proximal forefoot decreases. 
In this paper, we present an energy harvesting system to generate electrical energy from human foot strike. This result is achieved by integrating the piezoelectric material into the insole of a shoes and attaching the harvesting circuit on our calf. The electrical energy produced by different types of piezoelectric material and different circuit configurations of piezoelectric material is investigated. The effect of three factors, which are human body weight, human gender and human walking speed on the production of electrical energy are also studied.

\section{MATERIALS AND METHODS}

This section presents and explains the methodology on the investigation of human kinetic energy harvesting from human foot strike. In this study, several components are used to build a harvester such piezoelectric material, single phase full wave bridge rectifier, voltage regulator and rechargeable battery as illustrated in Figure 1.

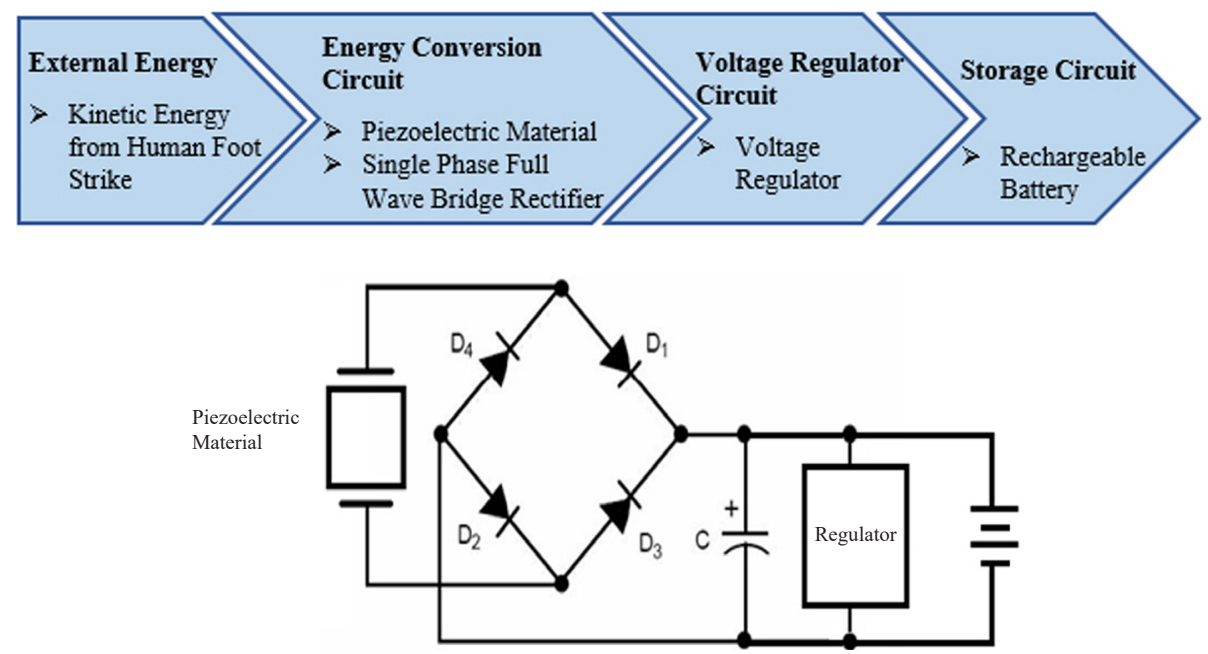

Figure 1: Block diagram of the harvester.

Since every foot strike produces certain amount of stress, this stress represents the kinetic energy generated by the foot strike. A PZT ceramic which can be used to convert the stress into an electrical energy is used as a transducer in this study. It is selected because it has a lighter weight and smaller size which is suitable to be installed into a shoes. Three types of PZT ceramic are chosen to be test and see which of it is suitable to be used in this harvester. Table 1 shows the details of the PZT piezoelectric ceramic. 
Table 1: Details of PZT piezoelectric ceramic.

\begin{tabular}{cccc}
\hline Type & Part Number & Diameter $(\mathrm{mm})$ & Thickness $(\mathrm{mm})$ \\
\hline A & MCFT-27T-4.2AL-127 & 27 & 0.45 \\
B & MCFT-31G-1.0A1-135 & 31 & 0.20 \\
C & MCFT-41T-1.0A1-141 & 41 & 0.22 \\
\hline
\end{tabular}

In this study, the $\mathrm{WC} 08 \mathrm{G}$ single phase full wave rectifier is used rather than build it by using four diodes to minimise the space. When the stress applied onto the PZT piezoelectric ceramic keep varying, the generated output voltage will also fluctuate. A voltage regulator is used in this study to regulate the voltage generated by the PZT piezoelectric ceramic into a constant voltage level which is suitable for battery charging. MAX666 voltage regulator is used in this study due to low power loss criteria. A rechargeable battery is used in this study to prove whether this harvester is suitable to be used to charge a battery. Since the output voltage of the voltage regulator is $5 \mathrm{~V}$ and the battery can only be charged when the charging voltage is slightly higher than the battery voltage, a $1.2 \mathrm{~V}$ AAA rechargeable battery with a capacity of $400 \mathrm{mAh}$ is used in this study.

Three types of PZT piezoelectric ceramic as mentioned in Table 1 are tested to see which of it can generate the greatest amount of electrical energy. Before the test is carried out, a machine which can be used to apply a periodic constant stress on the PZT piezoelectric ceramic as shown in Figure 2 is built. Since the electrical energy generated before and after the rectification need to be obtained in this stage, the terminal of the PZT piezoelectric ceramic must be connected to a single phase full wave bridge rectifier when taking the data of rectification signal.

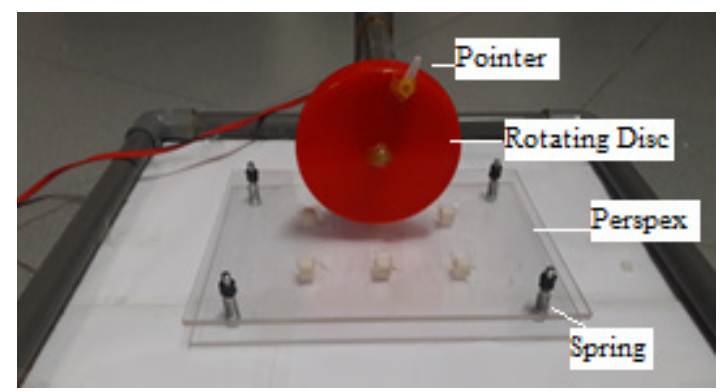

Figure 2: Machine which can apply a periodic constant stress.

In this test, the PZT piezoelectric ceramic is placed under the perspex. A power supply of $7 \mathrm{~V}$, which can apply around 16 times of stress in every $10 \mathrm{sec}$ is connected to the DC motor. When the motor starts to rotate, the white pointer 
hits on the perspex and a stress is applied onto the PZT piezoelectric ceramic. The output voltage of the PZT piezoelectric ceramics are observed and measured by using oscilloscope while the output current are measured by using a digital multimeter. The PZT piezoelectric ceramic which can generate the greatest amount of electrical energy is selected as a transducer in this study.

The next stage is to test different circuit configurations to see which circuit configuration can generate the greatest amount of electrical energy. The experiment is carried out by connecting the six pieces of PZT piezoelectric ceramics under four types of circuit configuration, which are in series, in parallel and in seriesparallel configuration as shown in Figure 3. The circuit configuration which can generate the greatest amount of electrical energy is selected to be used throughout this study.

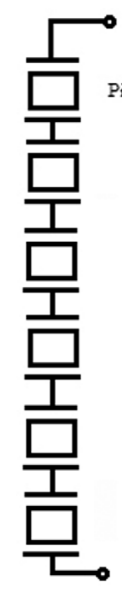

(a)

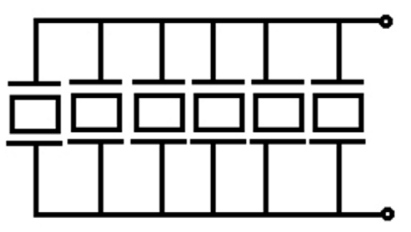

(b)

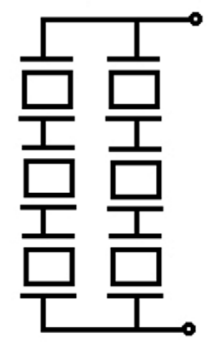

(c)

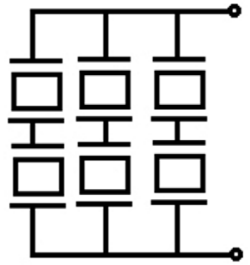

(d)

Figure 3: Schematic diagram of piezoelectric connected in different circuit configuration: (a) series, (b) parallel, (c) three series with two parallel and (d) two series with three parallel.

The next stage is to test the performance based on several factors, such as human body weight, human gender and human walking speed are carried out. The PZT piezoelectric ceramics are installed into the insole of the shoes as shown in Figure 4(a). In order to measure the output voltage, the output terminal of the harvesting circuit is connected to the Arduino Uno microcontroller to measure the voltage. The data is then transmitted to the computer via the Bluetooth module and a software, which is known as Coolterm to show and save the measured voltage. The harvesting circuit and the measuring circuit are put into a box and the box is tied at the bottom of the calf as shown in Figure 4(b). 
(a)

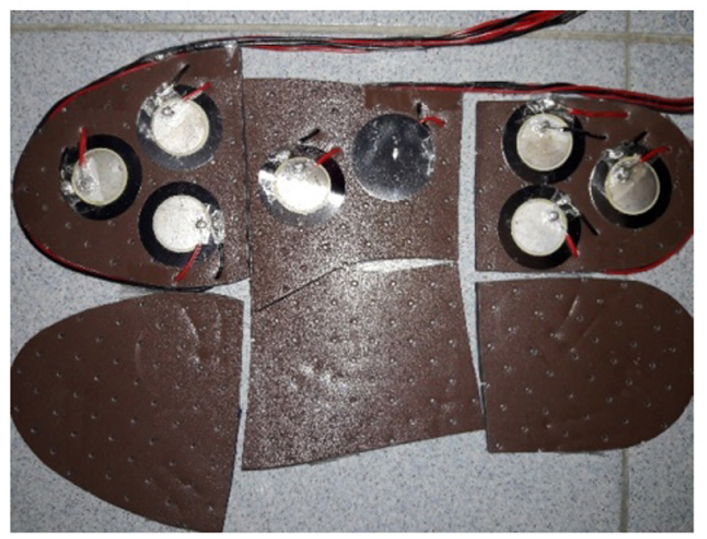

(b)

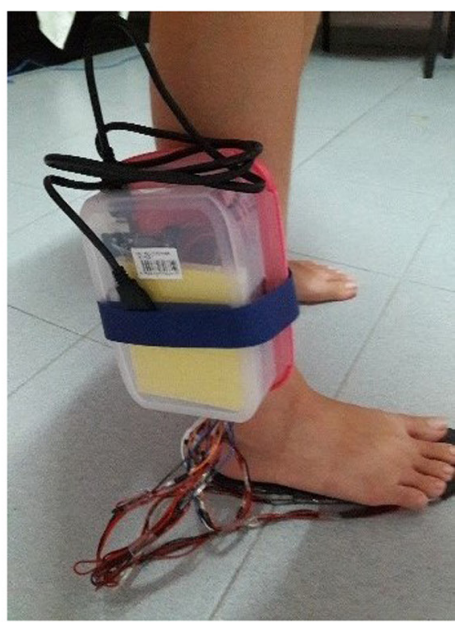

Figure 4: (a) PZT piezoelectric ceramics that installed into the insole of the shoes and (b) the harvesting circuit and the measuring circuit are put into a box.

A group of male and female with different body weight are selected to carry out the test. The body weight is divided into four categories, which are 40 to $49 \mathrm{~kg}, 50$ to $59 \mathrm{~kg}, 60$ to $69 \mathrm{~kg}$ and 70 to $79 \mathrm{~kg}$. Five candidates are selected for each of the category. However, due to there was limited number of female candidate in category of 70 to $79 \mathrm{~kg}$ and male candidate in category 40 to $49 \mathrm{~kg}$, only three candidates were selected for these two categories. Each of them were required to walk in four different speeds, which are $0.75 \mathrm{steps} / \mathrm{sec}$ (15 steps in 20 $\mathrm{sec}), 1.25 \mathrm{steps} / \mathrm{sec}(25 \mathrm{steps}$ in $20 \mathrm{sec}), 1.75 \mathrm{steps} / \mathrm{sec}(35 \mathrm{steps}$ in $20 \mathrm{sec})$ and $2.25 \mathrm{steps} / \mathrm{sec}(45 \mathrm{steps}$ in $20 \mathrm{sec})$. Table 2 shows the factors that need to be tested.

Table 2: Parameters to be tested in performance testing.

\begin{tabular}{ll}
\hline Factors & Details \\
\hline Human Body Weight & 40 to $49 \mathrm{~kg}$ \\
50 to $59 \mathrm{~kg}$ \\
60 to $69 \mathrm{~kg}$ \\
70 to $79 \mathrm{~kg}$ \\
Male \\
Human Gender & Female \\
& $0.75 \mathrm{steps} / \mathrm{sec}(15 \mathrm{steps}$ in $20 \mathrm{sec})$ \\
Human Walking Speed & $1.25 \mathrm{steps} / \mathrm{sec}(25 \mathrm{steps}$ in $20 \mathrm{sec})$ \\
& $1.75 \mathrm{steps} / \mathrm{sec}(35 \mathrm{steps}$ in $20 \mathrm{sec})$ \\
& $2.25 \mathrm{steps} / \mathrm{sec}(45 \mathrm{steps}$ in $20 \mathrm{sec})$ \\
\hline
\end{tabular}


In order to charge a battery, the charging voltage must be slightly greater than the battery voltage. However, due to the output voltage generated by the PZT piezoelectric ceramic fluctuates from 0 to around $20 \mathrm{~V}$ when stress is applied, a voltage regulator circuit is built in order to regulate the voltage into a constant voltage level which is suitable for battery charging. The schematic diagram of voltage regulator circuit is shown in Figure 5. This circuit is modified from the circuit done by the previous researcher. ${ }^{4}$ It can regulate the input voltage into a constant output voltage of $5 \mathrm{~V}$.

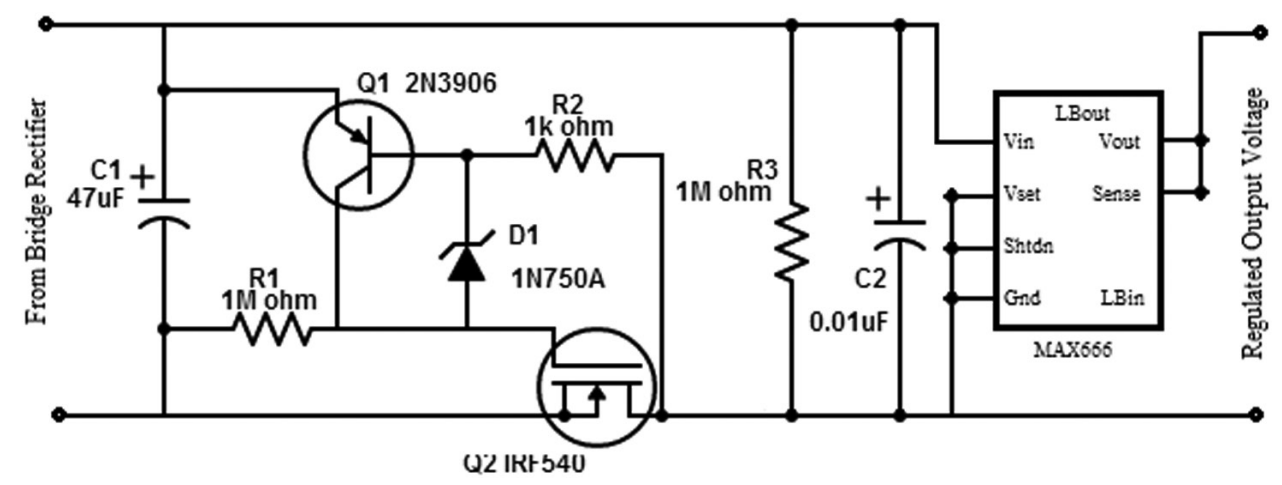

Figure 5: Schematic diagram of voltage regulator circuit.

In this circuit, the capacitor $\mathrm{C} 1$ is charged until its voltage is greater than the voltage between the emitter terminal and the base terminal of the 2N3906 PNP transistor plus the breakdown voltage of the Zener diode. Then, the 2N3906 PNP transistor turns on and causes the IRF540 N-channel Metal-Oxide-Semi Conductor Field-Effect Transistor (MOSFET) to turn on. This lead to the discharge of the capacitor $\mathrm{C} 1$ and hence the voltage of the capacitor $\mathrm{C} 1$ is regulated by the MAX666 voltage regulator to a voltage level of $5 \mathrm{~V}$. The output voltage of MAX666 decreases when the voltage across the capacitor $\mathrm{C} 1$ is less than $5 \mathrm{~V}$. When the capacitor $\mathrm{C} 1$ is fully discharged, the output voltage of MAX666 become $0 \mathrm{~V}$. At this condition, the capacitor $\mathrm{C} 1$ is recharged again. After the voltage regulator circuit is done, it is connected to a rechargeable battery to test whether it can be used to charge up the rechargeable battery.

\section{RESULTS AND DISCUSSIONS}

The results and the discussions are presented according to the experiment stages. 


\subsection{Preliminary Experimental Results of Different Types of Piezoelectric}

In the first stage of the experiment, three types of PZT piezoelectric ceramic which are different in diameter and thickness are compared. The electrical energy generated before and after the rectification of the $\mathrm{AC}$ signal are also presented. The results obtained are as shown in Figure 6.

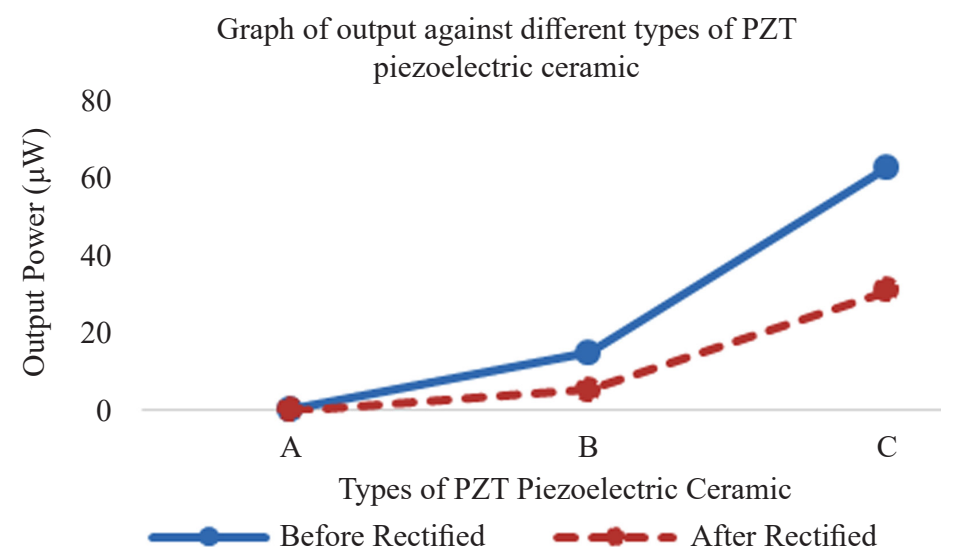

Figure 6: Comparison between the output power generated by different types of PZT piezoelectric ceramic before and after rectification.

The results showed that the electrical energy generated by different types of PZT piezoelectric ceramic increased from type A, type B, followed by type C. By referring to Table 1, the amount of electrical energy production increases with the applied stress area. This means that as the diameter increases, the applied stress area also increases, which leads to the increase in the amount of electrical energy generated by the PZT piezoelectric ceramic. Besides, a thinner PZT piezoelectric ceramic, which can be bend easily can also generate a greater amount of electrical energy. Based on the results obtained, although the type $\mathrm{C}$ piezoelectric ceramic can generate the greatest amount of electrical energy, it is too soft and can be break easily when a large stress is applied on it. Therefore, the type B, which has a smaller diameter and cannot be bend easily when compared to type $\mathrm{C}$ is selected as the transducer in this study.

The output voltage, current and power generated by the PZT piezoelectric ceramic before rectification is greater than after rectification. This is because during the rectification, there was a voltage drop across the single phase full wave bridge rectifier. This led to a power loss across the single phase full wave bridge rectifier and therefore the amount of electrical energy obtained after rectification was smaller when compared to before rectification. 


\subsection{Preliminary Experimental Results of Different Circuit Configurations}

In this experiment, the electrical energy generated by the PZT piezoelectric ceramics which are connected in four different types of circuit configurations as shown in Figure 3 are compared. Figure 7 shows the comparison between different circuit configurations of PZT piezoelectric ceramic. It shows the output voltage generated by the PZT piezoelectric ceramics is the greatest when they are connected in series while the output current generated by the PZT piezoelectric ceramics is the greatest when they are connected in parallel. This is because when the PZT piezoelectric ceramics were connected in series, the voltage generated by each of the PZT piezoelectric ceramics will be added up while in parallel configuration, the output current generated by each of the PZT piezoelectric ceramics will be added up. Since the output electrical energy generated by the series configuration is the greatest among the four types of circuit configurations, it is selected as the circuit configuration of the PZT piezoelectric ceramics in this study.

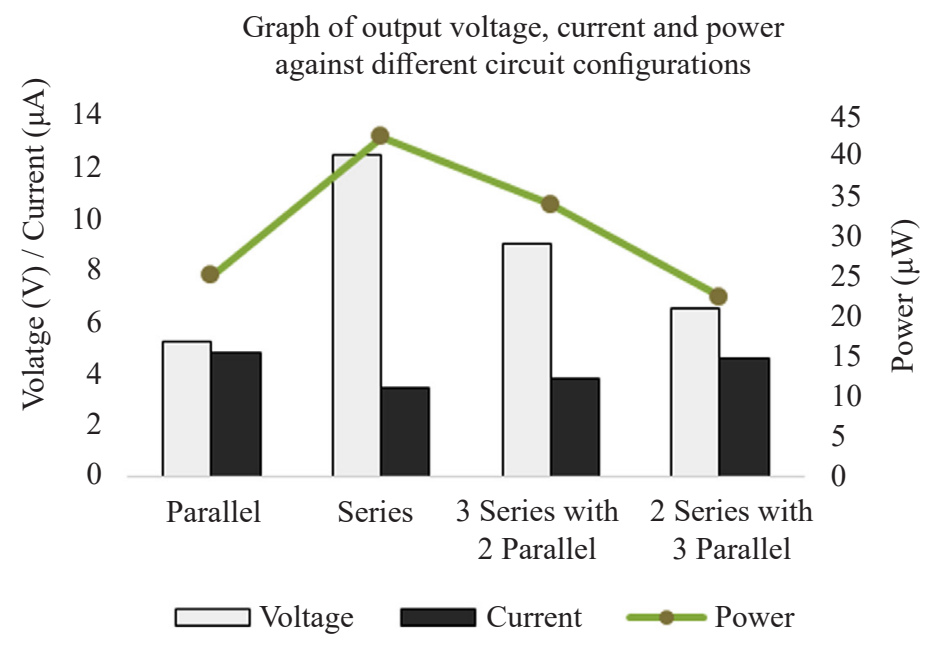

Figure 7: Comparison between results from different circuit configurations of PZT piezoelectric ceramic.

\subsection{Experimental Result of Performance Testing under Different Conditions}

This experiment is carried out in order to investigate the effect of human body weight, human gender and human walking speed on the amount of electrical energy production of the PZT piezoelectric ceramic. Figure 8 and 9 show the amount of electrical energy generated by male and female with different body weight under different walking speed respectively. 
Graph of average power against different human body weight under different walking speed

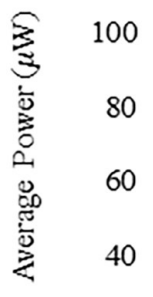

20
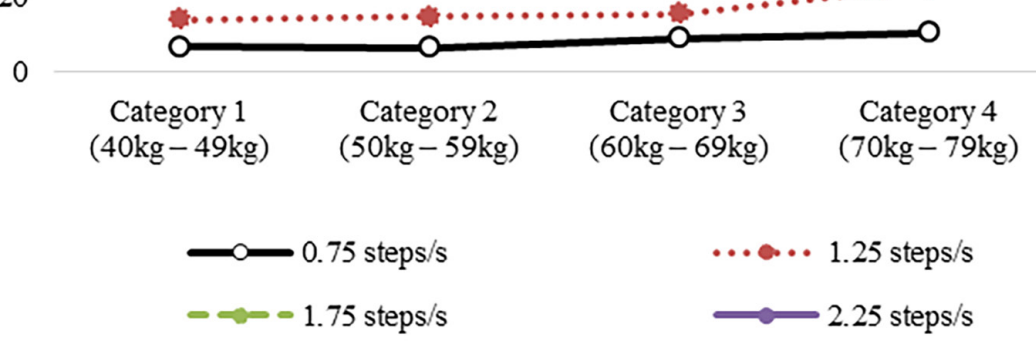

Figure 8: Amount of electrical energy generated by male with different body weight under different walking speed.

Graph of average power against different human body weight under different walking speed

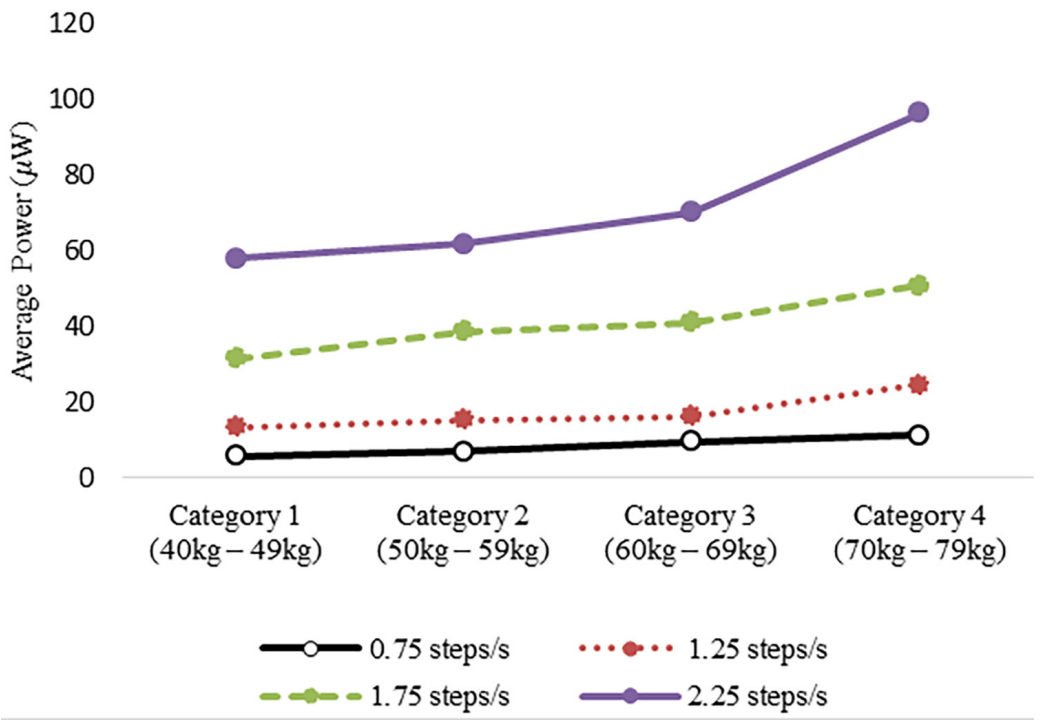

Figure 9: Amount of electrical energy generated by female with different body weight under different walking speed. 
Since the PZT piezoelectric ceramics are installed into the insole of a shoes in this study, the human body weight is used to represent the amount of stress applied on it. As discussed previously, people with greater body weight will have greater peak plantar pressure. This means that the greater the body weight, the greater the amount of stress applied onto the PZT piezoelectric ceramic and hence results in a greater amount of electrical energy production. Based on Figure 8 and 9, the results showed that as human body weight increases, the output power generated by the PZT piezoelectric ceramic also increases. This proved that the results obtained are the same as the expected results.

By referring to Section 1, it shows that the amount of electrical energy generated increases with the frequency of stress applied onto the piezoelectric. In this study, the frequency of stress applied is represented by human walking speed. The faster the walking speed, the higher the frequency of stress applied onto the piezoelectric, hence a greater amount of electrical energy is generated. Figure 8 and 9 show that the output power generated by the PZT piezoelectric ceramic increases with human walking speed. This proved that the results obtained from the experiment agree with the theoretical expectation.

In order to compare the amount of electrical energy generated by male and female, the line chart of the comparison between electrical energy generated by male and female under different body weight with a walking speed of 0.75 steps/ $\mathrm{sec}$ is constructed as shown in Figure 10. Since it is just to make the data to be easier to understood, only one set of data is presented.

Graph of average power against different human body weight with different gender at $0.75 \mathrm{steps} / \mathrm{sec}$ of walking speed

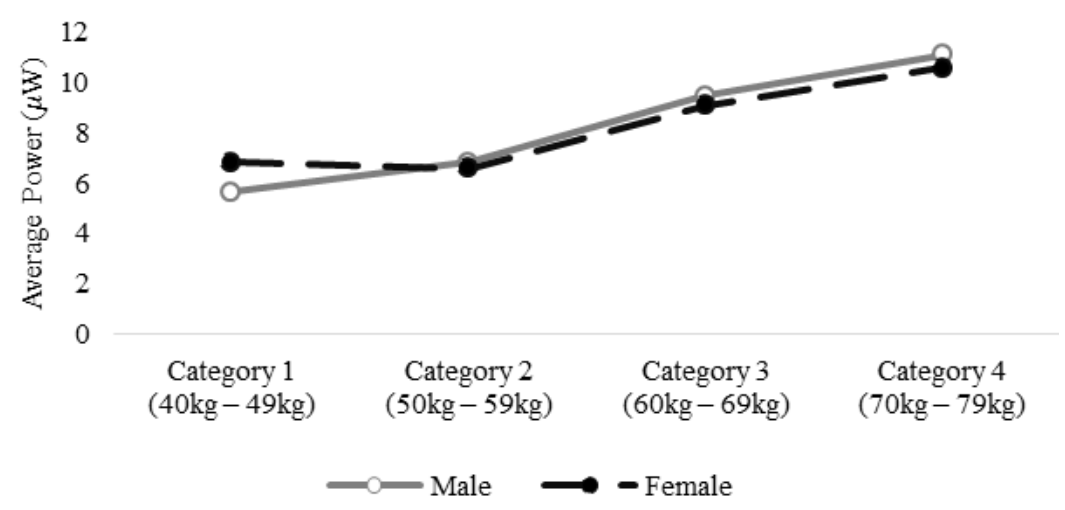

Figure 10: Comparison between electrical energy generated by male and female at a walking speed of $0.75 \mathrm{steps} / \mathrm{s}$. 
From the literature review in Section 1, it stated that the foot contact area of male is greater than the female. This implies that a male can apply a stress on a greater area when compared to a female. Since the amount of electrical energy generated increases with the applied stress area, this means that the electrical energy generated by a male is greater than a female. From Figure 10, it shows that the electrical energy generated by the male was slightly greater than the female. The results meet the expected results.

However, the electrical energy generated by the male is slightly smaller than the female in Category 1. This is because there is a female candidate who have applied heavy steps onto the piezoelectric. This results in extremely large amount of electrical energy to be generated and hence causes the average power generated by the female to be greater than the male in Category 1 . This can be conclude that the electrical energy generated by the PZT piezoelectric ceramic not only affected by the body weight, walking speed and gender, it also can be affected by the heavy step or lighter step applied.

One of the objectives of this study is to charge up a rechargeable battery by using the electrical energy harvested. However, by referring to Figure 8 and 9 , the electrical energy generated by the harvester is in the order of micro watts only, which is too small to be used for charging purpose. The current generated by the harvester are also in the order of micro amperes. This means that it needs to take quite a long time to fully charge up a battery with a capacity of milliampere hour. The time taken required to fully charge up a battery with a capacity of $400 \mathrm{mAh}$ is $42,417.8$ hours. This means that the harvester in this study is not suitable to be used to charge up a battery.

Although the electrical energy generated by the harvester is quite low, it is still able to power up a Light Emitting Diode (LED). To light up a LED, there are two conditions must be met. First, the voltage supply must be greater than the voltage drop across a LED, which is around $2.45 \mathrm{~V}$. Second, there must be a current pass through it regardless of how much the current is. Therefore, a LED can be light up when the capacitor used in the harvesting circuit is charged until it exceeds $2.45 \mathrm{~V}$. Since a LED can be lighted up by this harvester, this means that this harvester can also be used to power up a low power device.

\section{CONCLUSION}

The aim of this research is to investigate whether the electrical energy harvested from the kinetic energy produced by human foot strike is suitable to be 
used as a renewable energy source. Based on the results obtained, the MCFT-31G1.0A1-135 PZT piezoelectric ceramics is the most suitable transducer that can be used in this harvester. By connecting these PZT piezoelectric ceramics together in a series circuit configuration, the greatest amount of electrical energy can be generated. The results of the performance testing concludes that the male with a greater body weight who walk at a faster speed can generate the greatest amount of electrical energy, which is $96.25 \mu \mathrm{W}$ in this study. This amount of electrical energy could not be used to charge up a battery, but it can light up a LED. This result means that the electrical energy harvested is suitable to be used as renewable energy source for low power devices.

\section{REFERENCES}

1. Colorado River Commission of Nevada. (2002). World fossil fuels reserves and expected consumption. Las Vegas, NV: Colorado River Commission of Nevada (CRC).

2. U.S. Energy Information Administration. (2016). Renewable energy. Washington DC: U.S. Energy Information Administration. Retrieved from https://www.eia.gov/energyexplained/?page=renewable_home on 13 February 2017.

3. Tudor-Locke, C. et al. (2004). Descriptive epidemiology of pedometerdetermined physical activity. Med. Sci. Sports Exerc., 36(9), 1567-1573, https://doi.org/10.1249/01.MSS.0000139806.53824.2E.

4. Shenck, N. S. \& Paradiso, J. A. (2001). Energy scavenging with shoemounted piezoelectrics. IEEE Micro, 21(3): 30-42.

5. Xie, L. \& Cai, M. (2014). Increased piezoelectric energy harvesting from human footstep motion by using an amplification mechanism. Appl. Phys. Lett., 105(14), 1-5, https://doi.org/10.1063/1.4897624 .

6. Ramadan, K. S., Sameoto, D. \& Evoy, S. (2014). A review of piezoelectric polymers as functional materials for electromechanical transducers. Smart Mater. Struct., 23(3), 33001, https://doi.org/10.1088/09641726/23/3/033001.

7. Okayasu, M. \& Watanabe, K. (2015). The electric power generation characteristics of a lead zirconate titanate piezoelectric ceramic under various cyclic loading conditions. Ceram. Int., 41, 15097-15102, https://doi.org/10.1016/j.ceramint.2015.08.076.

8. Deepashini, H. et al. (2014). An insight into the plantar pressure distribution of the foot in clinical practice: Narrative review. Polish Ann. Med., 21(1), 51-56, https://doi.org/10.1016/j.poamed.2014.03.003. 